\title{
Subquadratic Approximation Algorithms for Clustering Problems in High Dimensional Spaces*
}

\author{
ALLAN BORODIN \\ Department of Computer Science, University of Toronto, Toronto, Canada M5S 3G4 \\ RAFAIL OSTROVSKY \\ Computer Science Department, University of California, Los Angeles, CA 90095 \\ YUVAL RABANI \\ Computer Science Department, Technion IIT, Haifa, Israel 32000
}

bor@cs.toronto.edu

rabani@cs.technion.ac.il

Editors: Nina Mishra and Rajeev Motwani

\begin{abstract}
One of the central problems in information retrieval, data mining, computational biology, statistical analysis, computer vision, geographic analysis, pattern recognition, distributed protocols is the question of classification of data according to some clustering rule. Often the data is noisy and even approximate classification is of extreme importance. The difficulty of such classification stems from the fact that usually the data has many incomparable attributes, and often results in the question of clustering problems in high dimensional spaces. Since they require measuring distance between every pair of data points, standard algorithms for computing the exact clustering solutions use quadratic or "nearly quadratic" running time; i.e., $O\left(d n^{2-\alpha(d)}\right)$ time where $n$ is the number of data points, $d$ is the dimension of the space and $\alpha(d)$ approaches 0 as $d$ grows. In this paper, we show (for three fairly natural clustering rules) that computing an approximate solution can be done much more efficiently. More specifically, for agglomerative clustering (used, for example, in the Alta Vista ${ }^{\mathrm{TM}}$ search engine), for the clustering defined by sparse partitions, and for a clustering based on minimum spanning trees we derive randomized $(1+\epsilon)$ approximation algorithms with running times $\tilde{O}\left(d^{2} n^{2-\gamma}\right)$ where $\gamma>0$ depends only on the approximation parameter $\epsilon$ and is independent of the dimension $d$.
\end{abstract}

Keywords: graph-theoretic clustering, high dimensional spaces, sparse partitions

\section{Introduction}

Clustering of data is an essential ingredient in many information retrieval systems (e.g., for building and maintaining taxonomies), and plays a central role in statistics, pattern recognition, biology, web search engines, distributed networks, and other fields. Recently, the concept of clustering has taken on some added significance as researchers have begun to view "data mining" as a question of finding "hidden clusters" in large collections of data.

*An extended abstract of this paper appeared in STOC 1999.

${ }^{\dagger}$ Work supported by BSF grant 96-00402, by MoS contract number 9480198, and by a grant from the Fund for the Promotion of Research at the Technion. 
(For a survey of clustering methods see (O'Rourke \& Toussaint, 1997; Eppstein, 1998) and references therein.) Informally, clustering algorithms attempt to form groups of similar objects into clusters based on the attributes of these objects. The question as to how best to define "the clustering problem" seems to be particularly difficult in large unstructured databases whose members are viewed as points in some high dimensional vector space.

A large class of clustering algorithms can be formulated within a graph-theoretic framework (see, for example, Chapter 10 of Duda, Hart, and Stork (2001)). In this formulation, the main ingredient of graph-theoretic clustering can be stated as follows: given $n$ data points in some metric space, do the following: (1) compute some spanning graph (such as the complete graph, or a minimum spanning tree) of the original data set; (2) Delete (in parallel) some edges of this graph (according to some criterion, such as distance); (3) output clustering (such as connected components or some partitioning of the nodes which depends on the topology) of the resulting graph.

For a more concrete example, consider the framework of hierarchical clustering where data points are joined into sets of objects, called clusters, with the property that any two sets are either disjoint or nested. In the agglomerative approach to hierarchical clustering, clusters are joined to form larger clusters based on the distance between the clusters. That is, all clusters with inter-cluster distance below a certain threshold are joined to form bigger clusters and so on. The distance between clusters can be defined in a number of ways. A simple and common choice is to compute the distance between the centroids of the clusters. Examples for applications that use such clustering rules include methods for determining consensus in biological sequencing data, and the mutual fragments heuristic for computing traveling salesman tours, see Eppstein (1998). (A more general choice of distance function is the so-called single-linkage distance, where the distance between clusters is the minimum distance between a point in one cluster and a point in the other cluster. Of course, measuring the distance between centroids, as well as other choices for a distance function, can be formulated as a single-linkage distance between cluster representatives; i.e., sets of points that replace the clusters.)

In this formulation, the core task for computing an agglomerative clustering is the following: Start with a complete weighted graph on the data set (points, or cluster representatives). Delete edges whose weight exceeds some given absolute bound, then output the connected components of the resulting graph. In addition to the afore-mentioned application to hierarchical clustering, this graph-theoretic clustering is used by the Alta Vista ${ }^{\mathrm{TM}}$ search engine in order to prune identical documents from its world-wide web database (Broder et al., 1997). We shall refer to it, somewhat imprecisely, as agglomerative clustering.

A second type of clustering with many applications in distributed computing (e.g., for routing tables, load balancing, file allocation) is defined by sparse partitions (Awerbuch \& Peleg, 1990). Roughly speaking, in our setting it says that given $n$ points and a distance $r$, define a graph where two points are adjacent if they are at (weighted) distance $r$ or less. Nodes must be partitioned into a collection of possibly overlapping clusters. The constraints on the clusters are that (i) the (unweighted graph theoretic) diameter of each cluster is small (a typical value is $O(\log n)$ edges where each edge corresponds to a distance of at most $r$ ); (ii) the clusters belong to a small number (again a typical number is $O(\log n)$ ) of color classes, so that the clusters within each class are disjoint and, (iii) for every point $x, x$ and all 
its neighbors are contained entirely in at least one cluster. Notice that this type of clustering is not hierarchical, as clusters (from different classes) may overlap.

A third type of clustering requires computing a minimum spanning tree (MST), so we call it MST-clustering. The clusters are the connected components of the forest that results from eliminating edges in the MST whose length exceeds a given parameter. Exact MSTclustering is equivalent to exact agglomerative clustering. It is not hard to see, however, that the approximate versions may differ substantially (because the approximation of MST is with respect to the total length of edges, and not with respect to the length of each edge separately). We remark that MST can be used as a subroutine for a variety of clustering methods.

We study all these clustering problems for points in $d$ dimensional Euclidean space. (We remark that our results easily extend to other spaces and norms such as the Hamming cube and the $L_{1}$ norm on the $d$-dimensional real space.) This is a natural setting for applications to information retrieval and data mining. The main issue we address is the following: A naive approach for any one of these problems (in high dimension) is to compute the distances among all pairs of points, and then execute any of the above clustering rules on the resulting graph. Of course, this naive approach has quadratic (in $n$ ) or worse time complexity. Can this quadratic behavior be avoided? This is the context in which similar problems, such as minimum spanning tree or closest pair, have been studied in computational geometry (see Goodman \& O'Rourke, 1997), but often the solutions are better than quadratic for low dimensions only. For example, Yao (1982) shows that MST can be computed in subquadratic time but the exponent is rapidly converging to 2 as the dimension grows. However, recent work on another geometric problem — nearest neighbor search (NNS)—shows that performance degradation as the dimension increases can be avoided if an approximation to the distances is allowed (Kleinberg, 1997; Indyk \& Motwani, 1998; Kushilevitz, Ostrovsky, and Rabani, 1998).

We therefore focus on approximate versions of the agglomerative and MST clustering problems. In most applications, the choice of both distances and the clustering rule is done on a heuristic basis, so approximate clustering is quite sufficient. The problem of sparse partition clustering already contains a certain degree of approximation implicit in the use of the "big Oh" notation and our methods can be used to derive improved bounds for the "exact problem" as defined with the understanding that the "big Oh" hides a factor of $1+\epsilon$. (For uniformity, we refer to our sparse partition algorithm as an approximation algorithm.) For all of the above problems and for any (approximation factor) $\epsilon$, we derive clustering algorithms with time complexity $\tilde{O}\left(d^{2} n^{2-\gamma}\right)$ for $\gamma>0$ which depends only on $\epsilon$. (The $\tilde{O}$ notation also hides factors of $\log n$.) Our subquadratic ${ }^{1}$ algorithms use some of the recent results on approximate NNS. In particular, we use modified versions of the algorithms and data structures of Kushilevitz, Ostrovsky, and Rabani (1998). One of the difficulties in obtaining our results is that these NNS algorithms are randomized, and their probabilistic guarantees are not strong enough to allow a simple high-probability successful termination of the clustering algorithms (without requiring quadratic or worse running time). Subsequent to our work, Indyk, Motwani, and Har-Peled (2002) have recently reported a $1+\epsilon$ approximation to MST with running time bounds very similar to ours (i.e., the same $\tilde{O}\left(d^{2} n^{2-\gamma}\right)$ for $\gamma>0$ but with slightly better functional dependence of $\gamma$ on $\epsilon$.) 
Once we are willing to settle for approximate solutions we can appeal to the dimension reduction techniques of Johnson and Lindenstrauss (1984) (see also Frankl \& Maehara, 1988; Linial, London, \& Rabinovich, 1995; Indyk \& Motwani, 1998). Simply stated, any $n$ points in Euclidean space of any dimension $d$ can be probabilistically mapped to an $O\left(\log n / \epsilon^{2}\right)$ dimensional space so that no distance is increased and (with high probability) no distance shrinks by more than a $1+\epsilon$ factor and this mapping has time complexity $O\left(d n \log n / \epsilon^{2}\right)$. It follows that for all the approximate clustering algorithms we consider, we can assume that $d=O(\log n)$. However, for the sake of completeness (and because the dependence on $d$ is interesting even for "small" $d$ ), we state all results in terms of $d$ and $n$ as well as the approximation factor $\epsilon$.

The naive approach to clustering in Euclidean space is to first compute all $\left(\begin{array}{c}n \\ 2\end{array}\right)$ distances, and then apply a clustering algorithm to the resulting weighted complete graph. For agglomerative clustering, (with standard implementations of a connected components algorithm) this naive approach would require $O\left(n^{2}\right)$ time. Broder (1997) give a heuristic used in Alta $\mathrm{Vista}^{\mathrm{TM}}$ that seems to give good running time in practice, but requires quadratic time in the worst case.

For sparse partitions (Awerbuch \& Peleg, 1990) give a polynomial time algorithm (in the number of edges). In the naive approach, we have a weighted complete graph, where all $\left(\begin{array}{l}n \\ 2\end{array}\right)$ edges are present. The following bounds are stated for this setting. Linial and Saks (1991) yield a randomized $\tilde{O}\left(n^{2}\right)$ time algorithm (where the $\tilde{O}$ notation hides polylog $(n)$ factors). The best result to date for the above setting (on a complete graph) is the deterministic $\tilde{O}\left(n^{2}\right)$ algorithm of Awerbuch et al. (1993).

The bottleneck for MST-clustering is computing the MST. We show how to apply the agglomerative clustering algorithm to derive a subquadratic approximate Euclidean minimum spanning tree (MST) algorithm. Sollin (see in Berge \& Ghouila-Houri, 1965) shows how to reduce the MST problem to a problem similar to exact NNS. Yao (1982) gives another reduction, and uses it to give a subquadratic algorithm for (exact) MST in low dimension. (Yao mentions obtaining a fast approximate MST algorithm as an open problem.) Yao's reduction seems specific to exact NNS, whereas Sollin's may be adapted to approximate NNS, though it is not clear how to overcome the probabilistic guarantees problem without resorting to our methods. The reduction of Yao yields an MST algorithm with time complexity $O\left(n^{2-2^{-d+1}}(\log n)^{1-2^{-d+1}}\right)$. (This is somewhat better than what would result from using Yao's algorithm with Sollin's reduction.) Chazelle (1985) improves this bound further, still the bound approaches quadratic behavior as the dimension grows. Notice that dimension reduction techniques cannot guarantee low distortion unless the dimension reduces to $\Omega(\log n)$, for which Chazelle's algorithm is still quadratic.

We also briefly indicate some other applications of our methods and algorithms to traditional computational geometry problems, for example a subquadratic algorithm for the Euclidean closest and furthest pair problem. The closest pair problem has many applications; for a full history (see Cohen \& Lewis, 1997; Eppstein, 1998) and references therein. Kleinberg (1997) uses his approximate NNS results to get an approximate closest pair algorithm with $O\left(\left(n^{2}+d n \log n\right) / \epsilon^{2}\right)$ running time (thus eliminating the dependency on the dimension for $d \leq n / \log n$ ). We show how to solve approximate closest pair (and approximate furthest pair) problems in subquadratic time for all $d$. 


\section{Preliminaries}

We denote by $E^{d}$ the $d$ dimensional Euclidean space; i.e., $\mathbb{R}^{d}$ with the metric induced by the $L_{2}$ norm.

An $\epsilon$-approximate $\Delta$-proximity table for a finite set $P \subseteq E^{d}$ is a data structure supporting the following operations

- $\operatorname{CONSTRUCT}(P)$, which creates a new instance of the data structure for $P \subseteq E^{d}$; this data structure consists of $|P|^{O(1)}$ entries, where $O(1)$ may depend on $\epsilon$. Each entry consists of a set of points.

- NEIGHBORS $(x)$, for any $x \in E^{d}$, which returns one of the above entries containing all the points in $P$ within distance $\Delta$ of $x$, and perhaps some additional points of $P$ within distance $(1+\epsilon) \Delta$ of $x$. (Notice that there may be several possible correct answers to NEIGHBORS.)

An efficient construction of an $\epsilon$-approximate $\Delta$-proximity table lies at the heart of our results. We can get such a construction by adapting the approximate nearest neighbor search algorithm of Kushilevitz, Ostrovsky, and Rabani (1998). The basic idea in that paper is that the Euclidean case can be embedded nearly isometrically in the Hamming cube. (In fact, as the dimension of the cube has to be similar to the dimension of the original space, only distances close to a given parameter $\Delta$ will be preserved.) In the cube, the dimension can be reduced to $O(\log |P|)$ using random linear transformations, preserving distances close to a given parameter. A single application of this construction would give a polynomially small (in $|P|$ ) error probability for any specific query. Applying this construction provides the following guarantee:

Lemma 1. For every $\beta>0$, there exists $c=c(\beta)>0$ such that for every $\epsilon>0$ there is a randomized implementation of an $\epsilon$-approximate $\Delta$-proximity table with the following properties:

1. CONSTRUCT takes $T(|P|)=|P|^{c / \epsilon^{2}}$ arithmetic operations;

2. NEIGHBORS takes $q(|P|)=c \epsilon^{-2} d^{2} \log |P|$ operations;

3. for any $x \in E^{d}$, the probability that an entry, and hence $\operatorname{NEIGHBORS}(x)$, returns an incorrect list is at most $|P|^{-\beta}$.

We also need to apply frequently a union/find algorithm. For our purposes it is sufficient to assume simply (say using balanced trees) that any union or find operation in a universe of $n$ elements can be performed within $g(n)=O(\log n)$ steps.

We also need to call approximate nearest (and furthest) neighbor algorithms ${ }^{2}$ of Kushilevitz, Ostrovsky, and Rabani (1998). These algorithms work for all queries and all distances. In particular, an $\epsilon$-ANN/ $\epsilon$-AFN table for a finite set $P \subseteq E^{d}$ is a data structure supporting the following operations

- CONSTRUCT-ANN/CONSTRUCT-AFN $(P)$, which creates a new instance of the data structure for $P \subseteq E^{d}$; this data structure consists of $|P|^{O(1)}$ "entries", where $O(1)$ may depend 
on $\epsilon$, and every entry contains either a single element of $P$ or a symbol indicating that the entry is empty.

- CLOSEST( $x$ ), for any $x \in E^{d}$, which returns a point in $P$ (contained in one of the entries), whose distance from $x$ is at most $1+\epsilon$ times the minimum distance of a point in $P$ from $x$.

- FURThest $(x)$, for any $x \in E^{d}$, which returns a point in $P$ (contained in one of the entries), whose distance from $x$ is at least $1-\epsilon$ times the maximum distance of a point in $P$ from $x$.

An efficient construction of an $\epsilon$-ANN/ $\epsilon$-AFN table is easily constructed from the approximate nearest neighbor search algorithm of Kushilevitz, Ostrovsky, and Rabani (1998). These results provide the following guarantee:

Lemma 2. For every $\beta>0$, there exists $c=c(\beta)$ such that for every $\epsilon>0$ there exists a randomized implementation of an $\epsilon-A N N / \epsilon-A F N$ table with the following properties:

1. CONSTRUCT-ANN/CONSTRUCT-AFN takes $T(|P|)=|P|^{c / \epsilon^{2}}$ arithmetic operations;

2. CLOSEST takes $\tilde{O}\left(\epsilon^{-2} d^{2}\right)$ operations;

3. FURTHEST takes $\tilde{O}\left(\epsilon^{-2} d^{2}\right)$ operations;

4. for any $x \in E^{d}$, the probability that CLOSEST ( $\left.x\right)$ or FURTHEST ( $x$ ) returns an incorrect answer is at most $|P|^{-\beta}$.

\section{Agglomerative clustering}

In this section we discuss the following clustering problem: Given a set $P \subseteq E^{d}$ of $n$ points and $\Delta>0$, partition $P$ into the connected components of the following graph $G_{P, \Delta}$. The graph $G_{P, \Delta}$ has node set $P$ and an edge connecting every pair of nodes at Euclidean distance $\Delta$ or less. In the approximate version of the problem, we are given an additional parameter $\epsilon$, and the graph $G_{P, \Delta}$ is replaced by a graph $G_{P, \Delta, \epsilon}$. The graph $G_{P, \Delta, \epsilon}$ has the same node set as $G_{P, \Delta}$. Its edge set contains all the edges of $G_{P, \Delta}$. In addition, it may contain edges connecting pairs of nodes at distance greater than $\Delta$, but no greater than $(1+\epsilon) \Delta$. (Notice that the choice of $G_{P, \Delta, \epsilon}$ is not necessarily unique-any such graph gives a correct solution to the approximate problem.) We remark that in addition to separating the graph into connected components, our algorithm can be easily modified to output a witness spanning tree for each component.

\subsection{The algorithm}

The main idea of the algorithm is to arbitrarily partition the set of points into subsets of size $n^{\delta}$ for some sufficiently small $\delta$. In doing so, we are able to generate a nearest neighbor structure for each such set in sublinear time. Hence the total time for generating all of the required nearest neighbor structures is subquadratic. For each point in the data set, we will find its neighbors in each structure and incrementally generate a graph (with a subquadratic number of edges) connecting neighbors. In order to insure that we can find a sufficient set of 


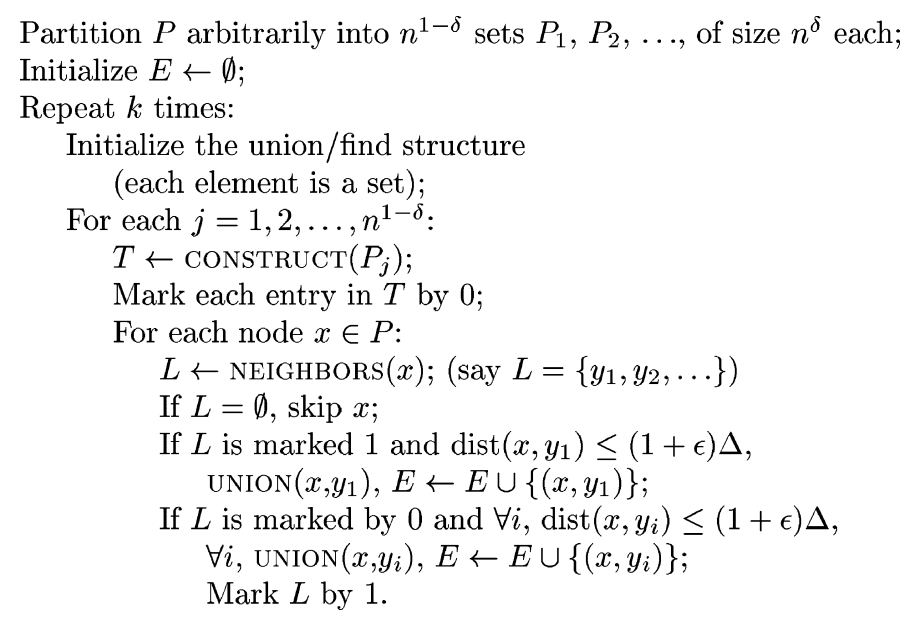

Figure 1. The agglomerative clustering algorithm.

edges in subquadratic time, we need to mark the entries in each structure that have already been used (so that the points in these entries are scanned at most once). We repeat the nearest neighbor searching process several times so as to boost the success probability. We are computing a sparse multigraph $G(P, E)$ whose connected components are the connected components of one of the desired graphs $G_{P, \Delta, \epsilon}$. We maintain a union/find structure with element set $P$ to construct these connected components. In addition, we maintain several proximity tables for subsets of $P$. We set $\beta=1$ in Lemma 1 and let $c>0$ be the constant guaranteed by Lemma 1 . Set $\delta=\epsilon^{2} / 2 c$ and $k=2 / \delta=4 c / \epsilon^{2}$.

The algorithm is shown in figure 1 . At the end of the algorithm, the desired partition of $P$ into clusters is the partition of the (sparse) graph $G$ constructed by the algorithm into its connected components.

\subsection{Notation}

Let $x \in \mathbb{R}^{d}$ and let $\ell>0$. Denote by $B(x, \ell)$ the closed ball around $x$ with radius $\ell$; i.e., the set $\left\{y \in \mathbb{R}^{d} \mid\|x-y\|_{2} \leq \ell\right\}$. Let $\overline{B(x, \ell)}$ denote the complement set.

\subsection{Correctness}

The correctness of the algorithm is an immediate corollary of the following claim:

Claim 3.

1. With high probability, (i.e., $\geq 1-n^{-\delta}$ ) every node $u \in P$ is in the same connected component as all the nodes in $B(u, \Delta)$. 
2. If $u, v \in P$ are in the same connected component of $G$, then there is a sequence of nodes $u=v_{0}, v_{1}, \ldots, v_{t}=v$, such that for all $i=1,2, \ldots, t, \operatorname{dist}\left(v_{i-1}, v_{i}\right) \leq(1+\epsilon) \Delta$.

Proof: To see (1), consider an $n^{\delta}$-subset $P_{i}$ of $P$ used by the algorithm and let $u$ be some point in $P$. Let $\mathcal{E}_{i}$ be the event that $u$ retrieves a proximity table entry containing all of $B(u, \Delta) \cap P_{i}$ and none of the points in $\overline{B(u,(1+\epsilon) \Delta)} \cap P_{i}$ (call such an entry good). $\operatorname{Pr}\left[\overline{\mathcal{E}_{i}}\right] \leq p^{k}$, where $p$ is the failure probability of a query. Thus $p \leq n^{-\delta}$, so $p^{k} \leq n^{-2}$. We have $n$ nodes and $n^{1-\delta}$ sets $P_{i}$, so with high probability every event $\mathcal{E}_{i}$ happens. Now, if $\mathcal{E}_{i}$ happens and $u$ retrieves a good entry marked by 0 , then we connect $u$ to all the nodes in that entry. Otherwise (the entry is marked 1), there is another node $v$ connected to all the nodes in the entry, and $u$ connects to one of the nodes of the entry.

For (2), use induction on the number of union operations performed. Consider a $\operatorname{UNION}(x, y)$ operation which joins two components $C_{1} \ni x, C_{2} \ni y$. The only case which does not follow from the induction hypothesis is $u \in C_{1}$ and $v \in C_{2}$. But by the induction hypothesis, there is a sequence $u=v_{0}, v_{1}, \ldots, v_{s}=x$, and another sequence $y=$ $v_{s+1}, v_{s+2}, \ldots, v_{t}=v$, such that for every $i \neq s+1,1 \leq i \leq t, \operatorname{dist}\left(v_{i-1}, v_{i}\right) \leq(1+\epsilon) \Delta$. By the specification of the algorithm, $\operatorname{dist}\left(v_{s}, v_{s+1}\right)=\operatorname{dist}(x, y) \leq(1+\epsilon) \Delta$.

\subsection{Analysis}

We analyze the complexity of one iteration of the outer "Repeat loop" and then multiply by $k$ to obtain the total running time. For simplicity we suppress the influence of $c$ and $\epsilon$ in the big $O h$ notation. The time complexity of the "Repeat loop" is upper bounded by the sum

$$
D+N+B+G+U
$$

where $D$ is the cost of building the $n^{1-\delta}$ proximity tables, $N$ is the minimum search cost for the nodes (i.e., assuming they retrieve entries marked 1 only), $B$ is the cost for retrieving bad entries, $G$ is the cost induced by retrievals of good 0 -marked entries, and $U$ is the cost of performing union/find. We analyze each term separately.

Claim 4. Let $\beta=1$ and let $c=c(\beta)$ be the constant from Lemma 1. Let $g(n)=O(\log n)$ be the worst case cost of a union/find algorithm. Then,

$$
\begin{aligned}
D & =n^{1-\delta} T\left(n^{\delta}\right)=o\left(n^{3 / 2}\right) \\
N & =n^{2-\delta}\left(q\left(n^{\delta}\right)+d\right)=O\left(d^{2} n^{2-\epsilon^{2} / 2 c} \log n\right) \\
\mathrm{E}[B] & =O\left(n^{2-\delta} d\right)=O\left(d n^{2-\epsilon^{2} / 2 c}\right) \\
G & =O\left(n T\left(n^{\delta}\right) d\right)=O\left(d n^{3 / 2}\right) \\
U & =g(n)\left(n^{2-\delta}+n T\left(n^{\delta}\right)\right)=O\left(n^{2-\epsilon^{2} / 2 c} \log n\right)
\end{aligned}
$$




\section{Proof:}

- For $D$ : In each iteration we have to construct $n^{1-\delta}$ proximity tables, each for $n^{\delta}$ points. The construction of each table takes $T\left(n^{\delta}\right)$ steps.

- For $N$ : In each iteration we process each of the $n$ points. Each point requires a search in $n^{1-\delta}$ proximity tables. The search in each proximity table takes $q\left(n^{\delta}\right)$ time. If the retrieved entry is not empty and is marked 1, there is an additional cost of $O(d)$ to compute the distance to the first point on the list.

- For $B$ : In each iteration we access $n^{1-\delta}$ proximity tables, searching for $n$ points in each table. For each table, for each point, the probability that the point retrieves a bad entry is at most $\left(n^{-\delta}\right)$. Therefore the expected number of bad entries handled in each proximity table is at most $n^{1-\delta}$. For each bad entry, we may have to compute the distance to all the points in the table. This costs $O\left(n^{\delta} d\right)$.

- For $G$ : In each iteration we handle $n^{1-\delta}$ proximity tables. Each table has at most $T\left(n^{\delta}\right)$ entries. An entry is accessed as a good entry that is marked 0 at most once. If this happens, we compute the distances to all the points listed, at most $n^{\delta}$. Each distance costs $O(d)$.

- For $U$ : The bound follows from an estimate on the total number of union operations performed. In each iteration we handle $n^{1-\delta}$ proximity table. Each proximity table is accessed by $n$ points. If a point retrieves an entry marked 1 , it causes at most one union operation, so there are at most $n$ such operations per table. Good entries marked 0 cause at most $n^{\delta}$ union operations. For each entry we do this at most once. Thus, the number of these union operations per table is at most $n^{\delta} T\left(n^{\delta}\right)$.

Theorem 5. The total running time of the agglomerative clustering algorithm is $O\left(d^{2} n^{2-\epsilon^{2} / 2 c} \log n\right)$.

\section{Sparse partitions}

In this section we discuss computing a sparse partition clustering of a finite set of points $P \subseteq E^{d}, P=\left\{x_{1}, \ldots, x_{n}\right\}$. Our definition of sparse partitions is the Euclidean analogue of the definitions given in Awerbuch and Peleg (1990), Awerbuch et al. (1993), and Linial and Saks (1991) for undirected graphs where in those papers distance refers to path length.

A sparse partition $\mathcal{C}$ of $P$ (with parameter $r>0$ ), is a collection of subsets (called clusters) $S_{1}, S_{2}, \ldots, S_{m} \subseteq P$ satisfying the following conditions:

1. The (Euclidean) diameter of each cluster $S_{i}$ is at most $O(r \log n)$.

2. For every $x \in P, P \cap B(x, r)$ is contained completely in at least one of the clusters where $B(x, r)$ is the Euclidean ball of radius $r$ centered at $x$.

3. The clusters can be grouped into $O(\log n)$ classes, so that the clusters in each class are disjoint. (It follows that for each $x \in P$, there are at most $O(\log n)$ clusters containing $x$.) Furthermore, the distance between any two clusters in the same class is greater than 
$r$; that is, if clusters $S_{i}$ and $S_{j}$ are in the same class with $x \in S_{i}$ and $y \in S_{j}$, then the Euclidean distance between $x$ and $y$ is greater than $r$.

(The original definition of sparse partitions is more general in that it allows tradeoffs between the values in conditions 1 and 3. The definition here uses the most common values in applications. Our results extend to these general tradeoffs.)

\subsection{The algorithm}

We first briefly describe the sparse partition algorithm in Linial and Saks (1991). Here the algorithm is given a undirected $n$-node graph $G$ and outputs $O(\log n)$ classes of clusters where each class is a partition of the node set, each edge is contained in at least one cluster and the diameter of each cluster is $O(\log n)$. Each class of clusters is created in an independent phase. In a phase, the following procedure is used to grow clusters. Pick a random integer $i \in\{1, \ldots, 2 \log n\}$ using the truncated geometric distribution where $\operatorname{Pr}[i=k]=2^{-k}$ for $1 \leq k \leq 2 \log n-2$ and $\operatorname{Pr}[i=2 \log n-1]=\operatorname{Pr}[i=2 \log n]=2^{-2 \log n+1}$. Then pick an arbitrary unclustered node $x$ and construct a cluster containing all unclustered nodes within (graph theoretic) distance $i$ from $x$. This process is repeated until all nodes have been clustered at which point the phase is completed.

In our geometric problem, the sparse partitioning algorithm can be applied to the graph $G_{P, r}$ where every edge is representing a Euclidean distance of at most $r$. Of course $G_{P, r}$ may have a quadratic number of edges and hence a direct application of the algorithm would require at least quadratic running time. We sped up the algorithm by implicitly using one of the graphs $G_{P, r, \epsilon}$. In fact, as in the previous section, we compute a sparse subgraph that is sufficient for computing the sparse partition. Using the $\epsilon$-approximate $r$-proximity tables we can find for an unclustered point $y$ a set $\tilde{B}(y, r)$ containing all unclustered points at distance at most $r$ from $y$ and possibly some unclustered points at distance at most $(1+\epsilon) r$ from $y$. A more formal description of the algorithm appears in figure 2 .

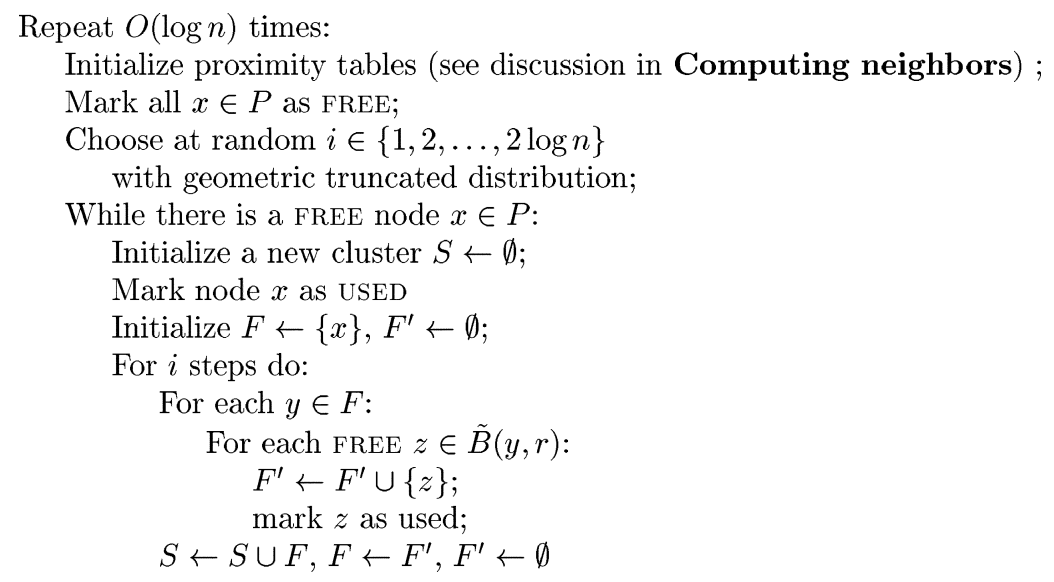

Figure 2. The sparse partitions algorithm. 


\subsection{Computing neighbors}

We compute the set $\tilde{B}(y, r)$ using the proximity tables that are initialized at the beginning of the phase. We first explain the initialization: Let $\delta$ and $k$ be as in the previous section. We partition $P$ into $n^{1-\delta}$ disjoint sets of size $n^{\delta}$ each. For each set we construct $k$ independent $\epsilon$-approximate $r$-proximity tables. We also mark all the entries in all the tables by 0 . This completes the initialization. We construct the set $\tilde{B}(y, r)$ as follows: We fetch NEIGHBORS $(y)$ in each of the $k n^{1-\delta}$ tables. Let $L_{1}, L_{2}, \ldots, L_{k n^{1-\delta}}$ be the resulting lists. We ignore all lists marked by 1 . For every list $L$ marked 0 we compute $\operatorname{dist}(y, z)$, for all $z \in L$. If any point in $L$ is further from $y$ than $(1+\epsilon) r$, we leave $L$ marked by 0 . Otherwise we add the elements of $L$ to $\tilde{B}(y, r)$ and mark $L$ by 1 .

\subsection{Correctness}

Here we prove that the above algorithm indeed produces the desired sparse partition clustering with high probability. The main issue is to show that $\tilde{B}(y, r)$ is computed correctly. The rest of the argument follows in general the line of argument in Linial and Saks (1991). The added difficulty is that because we have approximate distances only, proximity between points is no longer a symmetric property.

Claim 6. With probability ${ }^{3}$ at least $\frac{19}{20}$ the following holds for every $y \in P$ : If the algorithm computes $\tilde{B}(y, r)$ then this set contains all the points in $B(y, r)$ that are still FREE, and none of the points in $\overline{B(y,(1+\epsilon) r)}$.

Proof: The latter claim is obvious because the algorithm checks the distances to the points it adds to $\tilde{B}(y, r)$. To see the former claim, we argue as in the proof of Claim 4 that the probability that the good entries retrieved by $y$ do not contain all the points in $B(y, r)$ is at most $n^{-2}$, and therefore the probability that this happens for any $y$ is at most $n^{-1}$. Finally, notice that if an entry is marked 1 , then the status of all the points listed in the entry (in the current phase) is USED.

Claim 7. Consider any particular phase. Let $y \in P$ be the first point in $B(x, r)$ that is placed in $F$ in that phase. If $y$ is placed in $F$ just before the execution of iteration $j \leq 2 \log n-2$ of the "For" loop of that phase, then $B(x, r)$ is contained in a cluster generated in the phase with probability at least $\frac{1}{5}$.

Proof: Let $F_{j}$ be the set of points in $B(x, r)$ that are in $F$ at the start of iteration $j$; that is, if $j>1$ these are the points that were placed in $F^{\prime}$ during iteration $j-1$. Assume for the moment that $x \notin F_{j}$. Clearly, $y \in F_{j} \subseteq F$. Moreover, at the start of the $j$ th iteration, all the points in $B(x, r) \backslash F_{j}$ (and in particular $x$ ) are FREE. As the $j$ th iteration is executed, we know that $i \geq j$. Given that $i \geq j$, and that $j \leq 2 \log n-2$, the conditional probability that $i \geq j+2$ is $\frac{1}{4}$. So assume that the event $i \geq j+2$ holds, and further assume that the event in Claim 6 holds. This happens with probability at least $\frac{1}{4}-\frac{1}{20}=\frac{1}{5}$. As $y \in B(x, r)$, $\operatorname{dist}(y, x) \leq r$. Therefore, $x \in B(y, r) \subseteq \tilde{B}(y, r)$, and $x$ is placed in $F^{\prime}$ during the $j$ th 
iteration (by Claim 6). Let $F_{j+1}$ be the set of points in $B(x, r)$ that are placed in $F^{\prime}$ during the $j$ th iteration. At the end of the $j$ th iteration, all the points in $F_{j}$ are added to the cluster $S$, and all the points in $F^{\prime}$ are placed in $F$. In particular, $x$ is placed in $F$, and so are all the other points in $F_{j+1}$. We have assumed that $i \geq j+2$. Therefore iteration $j+1$ is executed. Notice that at the start of this iteration, the points in $B(x, r) \backslash\left(F_{j} \cup F_{j+1}\right)$ are FREE. Denote these points by $F_{j+2}$. As $x \in F$, during iteration $j+1$ the FREE points in $\tilde{B}(x, r)$ are placed in $F^{\prime}$. These include all the points of $F_{j+2}$. At the end of iteration $j+1$, the points in $F$, and in particular the points of $F_{j+1}$, are placed in the cluster $S$. The points in $F^{\prime}$, in particular all of $F_{j+2}$ are placed in $F$. As we are assuming that iteration $j+2$ is executed as well, at the end of that iteration the points of $F_{j+2}$ are placed in the cluster $S$. Because $F_{j} \cup F_{j+1} \cup F_{j+2}$ are all the points in $B(x, r)$, the claim follows. If $x \in F_{j}$ then a similar argument shows that $S$ will contain all the points in $B(x, r)$ with even larger probability.

Claim 8. Consider any particular phase. $\forall x$, the probability that the first $y \in B(x, r)$ to be clustered is reached in the last two iterations of the main loop for that phase is $\frac{4}{n^{2}}$.

Proof: $i$ is chosen once each phase, and the probability that $i>2 \log n-2$ is $\frac{4}{n^{2}}$.

The above claims imply

Theorem 9. With high probability the algorithm produces a sparse partitioning clustering with parameter $r$.

Proof: By Claims 7 and 8, for every point $x \in P$, the following holds. In any phase, the probability that $B(x, r) \cap P$ is completely contained in a cluster generated in that phase is a bit below $\frac{1}{5}$, but clearly more than, say $\frac{1}{6}$. Thus, after $O(\log n)$ phases, with high probability this property holds for all points $x$. The bound on the diameters of the clusters follows from the choice of $i$. The bound on the number of clusters containing a point follows from the fact that there are $O(\log n)$ iterations, and each iteration generates a collection of disjoint clusters.

\subsection{Analysis}

Let $\delta$ and $k$ be as described in the implementation. The time complexity for a phase in the above algorithm is (similar to agglomerative clustering) upper bounded by the sum

$$
D+N+B+G+U
$$

where $D$ is the cost of building the $k n^{1-\delta} \log n r$-proximity tables, $N$ is the minimum search cost for $\tilde{\mathcal{B}}(y, r)$ (i.e. cost of retrieving all the pointers only), $B$ is the cost for retrieving entries that are marked 0 , but which contain points further then $(1+\epsilon) r, G$ is the cost induced by retrievals of good 0-marked entries (i.e. such that all are at distance within $(1+\epsilon) r$, and $U$ is the cost of performing set unions. Again, we analyze each cost separately. 
Claim 10. Let $\beta=1$ and let $c=c(\beta)$ be the constant from Lemma 1. Then,

$$
\begin{aligned}
D & =o\left(k n^{3 / 2} \log n\right) \\
N & =O\left(k d^{2} n^{2-\delta} \log ^{2} n\right) \\
\mathrm{E}[B] & =O\left(k n^{2-\delta} d \log n\right) \\
G & =O\left(k n^{3 / 2} \log n\right) \\
U & =O\left(n^{2-\delta} \log ^{2} n\right) .
\end{aligned}
$$

Proof: Analogous to Lemma 4.

Theorem 11. The total running time of the sparse partitions algorithm is $O\left(d^{2} n^{2-\epsilon^{2} / 2 c}\right.$ $\left.\log ^{2} n\right)$.

\section{Computing an approximate MST and other related problems}

In this section we discuss several other geometric problems that can be approximated in subquadratic time using our methods. First, we consider the problems of computing a $1+\epsilon$ approximation to the closest and to the furthest pair of points, an application also considered by Kleinberg (1997). We have the following result:

Lemma 12. For each constant $\epsilon$ there exists $\delta>0$ (the same $\delta$ as in agglomerative clustering) so that in time $\tilde{O}\left(d^{2} n^{2-\delta}\right)$ we can compute w.h.p. a pair of points which are within a factor $1+\epsilon$ of the distance of the closest (or furthest) pair of points.

Proof: Partition the set $P$ into $n^{1-\delta}$ subsets of size $n^{\delta}$ each, as before. For each subset we build an $\epsilon$-ANN (or an $\epsilon$-AFN) data structure. We then search each of the structures for each point in $P$. By repeating the search several times and taking the best answer, we can reduce the error probability sufficiently.

We now consider the problem of computing an approximate minimum spanning tree (MST).

Lemma 13. For each constant $\epsilon$ there exists $\delta$ so that we can compute w.h.p. a $(1+\epsilon)$ approximation to the minimum spanning tree in time $\tilde{O}\left(d^{2} n^{2-\delta}\right)$.

Proof: Iterate over several computations of agglomerative clustering, each time reducing the parameter $\Delta$ by a factor of about $1+\epsilon$. Notice that our agglomerative clustering algorithm outputs a sparse graph $G$ as a witness to the connectivity of each cluster. We use such a witness here (i.e., a "black-box" agglomerative clustering algorithm that outputs the partition into clusters alone is insufficient). Whenever a cluster in one iteration is split in the next, we add to the growing forest edges connecting the current fragments of the cluster. These edges are taken from the graph $G$ computed in the previous iteration. (Notice that the 
graph in the current iteration may differ from the the graph in the previous iteration.) We add edges of $G$, that do not connect two points that are currently also in the same cluster, and do not close a cycle. (Because $G$ is sparse enough, we can check all its edges.)

We can begin with any $\Delta$ which on the one hand guarantees a single cluster, and on the other hand is not too big (close enough to a lower bound on the MST). For example, we can take $1+\epsilon$ times the result of an approximate furthest pair search. We can stop when $\Delta$ reaches a value small enough to allow us to take the remaining connections as we please. Because an MST contains $n-1$ edges, a value of about $\epsilon / n$ times the initial value of $\Delta$ is sufficient (recall that the initial value is close to a lower bound on the MST). Thus, the number of agglomerative clustering computations, for fixed $\epsilon$, is $O(\log n)$.

As mentioned previously, we can now use such an approximate MST and any threshold value $\theta$ as the basis for approximate MST clustering by deleting from the MST any edges of distance larger than $\theta$ and viewing the resulting components as clusters. We note that this MST clustering might be different from an approximate agglomerative clustering using the same parameter $\theta$.

\section{Acknowledgments}

We thank Andrei Broder for his motivating discussions regarding the Alta-Vista ${ }^{\mathrm{TM}}$ search engine.

\section{Notes}

1. Some papers will refer to any bound which is $o\left(n^{2}\right)$ as being "sublinear" since the naive presentation of the input would require $n^{2}$ distances.

2. The algorithms of Kushilevitz, Ostrovsky, and Rabani (1998) are easily adapted to finding furthest neighbors.

3. The choice of constant is arbitrary.

\section{References}

Awerbuch, B., \& Peleg, D. (1990). Sparse partitions. In Proc. of 21st FOCS (pp. 503-513).

Awerbuch, B., Berger, B., Cowen, L., \& Peleg, D. (1993). Near-linear cost sequental and distributed constructions of sparse neighborhood covers. In Proc. of 34th FOCS (pp. 639-647).

Berge, C., \& Ghouila-Houri, A. (1965). Programming, games, and transportation networks. John Wiley.

Broder, A., Glassman, S., Manasse, M., \& Zweig, G. (1997). Syntactic clustering of the Web. In Proceedings of the Sixth International World Wide Web Conference (pp. 391-404).

Cohen, E., \& Lewis, D. (1997). Approximating matrix multiplication for pattern recognition tasks. In Proc. of 8th SODA.

Chazelle, B. (1985). How to search in history. Information and Control, 64, 77-99.

Duda, D., Hart, P., \& Stork, D. (2001). Pattern classification (2nd edition). John Wiley and Sons.

Eppstein, D. (1998). Fast hierarchical clustering and other applications of dynamic closest pair. In Proc. of 9th SODA.

Frankl, P., \& Maehara, H. (1988). The Johnson-Lindenstrauss lemma and the sphericity of some graphs. J. of Combinatorial Theory B, 44, 355-362.

Goodman, J., \& O'Rourke, J. (Eds.) (1997). Handbook of discrete and computational geometry. CRS press. 
Indyk, P., \& Motwani, R. (1998). Approximate nearest neighbors: Towards removing the curse of dimensionality. In Proc. of 30th STOC.

Indyk, P., Motwani, R., \& Har-Peled, S. (2002). Private communication (including a complete version of Indyk \& Motwani, 1998).

Johnson, W.B., \& Lindenstrauss, J. (1984). Extensions of Lipschitz mappings into Hilbert space. Contemporary Mathematics, 26, 189-206.

Jaromczyk, J., \& Toussaint, G. (1992). Relative neighborhood graphs and their relatives. Proc. IEEE, 90, 15021517.

Kleinberg, J. (1997). Two algorithms for nearest-neighbor search in high dimensions. In Proc. of 29th STOC (pp. 599-608).

Kushilevitz, E., Ostrovsky, R., \& Rabani, Y. (1998). Efficient search for approximate nearest neighbor in high dimensional spaces. SIAM J. Comput., 30:2, 457-474. (Preliminary version in Proc. of 30th STOC, 614-623). Linial, N., London, E., \& Rabinovich, Y. (1995). The geometry of graphs and some of its algorithmic applications. Combinatorica, 15:2, 215-245.

Linial, N., \& Saks, M. (1991). Decomposing graphs into regions of small diameter. In Proc. of 2nd SODA (pp. 320-336).

O'Rourke, J., \& Toussaint, G. (1997). Pattern recognition. In Goodman \& O'Rourke (1997) (pp. 797-813).

Yao, A. C. (1982). On constructing minimum spanning trees in $k$-dimensional spaces and related problems. SIAM J. Comput., 11:4, 721-736.

Received December 16, 2002

Revised January 16, 2004

Accepted January 20, 2004

Final manuscript March 4, 2004 